\title{
Magnetohydrodynamics Free Convection Flow of Incompressible Fluids over Corrugated Vibrating Bottom Surface with Hall Currents and Heat and Mass Transfers
}

\author{
Vincent M. Bulinda $\mathbb{D}^{\mathbb{D}}$, Giterere P. Kang'ethe, and Phineas R. Kiogora \\ Department of Pure and Applied Mathematics, Jomo Kenyatta University of Agriculture and Technology, \\ Juja 62000-00200, Kenya \\ Correspondence should be addressed to Vincent M. Bulinda; vbulinda@kisiiuniversity.ac.ke
}

Received 15 November 2019; Accepted 13 January 2020; Published 1 March 2020

Academic Editor: Sazzad Hossien Chowdhury

Copyright ( $) 2020$ Vincent M. Bulinda et al. This is an open access article distributed under the Creative Commons Attribution License, which permits unrestricted use, distribution, and reproduction in any medium, provided the original work is properly cited.

Magnetohydrodynamics free convection flow of incompressible fluids over corrugated vibrating bottom surface with Hall currents and heat and mass transfers considering heat flux is discussed. The corrugation patterns suggested are sinusoidal in nature. The governing equations are solved by the explicit finite difference numerical method of the forward-time backward-space scheme to obtain the analytical results for velocity, concentration, and temperature profiles. The unsteady resultant velocities, concentration, and temperature for various values of physical parameters are discussed in detail, and it is shown that they have significant effects on the fluid flow, and heat and mass transfers are shown graphically.

\section{Introduction}

More advances in modern technology calls for the need to design processing equipment that complies with the economic, environmental, and energy saving with high efficiency of mass and heat transfers.

Corrugated structures are well known for heat transfer enhancement applied in various engineering problems such as heat exchangers, air-conditioning devices, refrigeration systems, chemical reactors, and fuel rod [1]. Corrugated channels can be designed with different corrugation angles with different arrangements of channel surface $[2,3]$. Corrugated ducts are designed in a way that fabricates heat [4] and improves heat transfer efficiency in an appropriate Reynolds number regime [5]. A numerical study on heat transfer of sinusoidal wavy channels at zero-degree phase shift was conducted by [6, 7]. Abo-Eldahab and Aziz [8] studied hydrodynamics and thermal behaviour of free connective flow over an isothermal, vertical flat plate and convective flow of heat-generating fluid past a vertical plate with Hall current and heat flux through a porous medium. Idowu et al. [9] studied the impact of heat and mass transfers on MHD oscillatory flow of Jeffery fluid in a porous channel with thermal conductivity, Dufour and Soret. 3D numerical predictions of fluid flow and heat transfer between crosscorrugated plates were studied in [10] and obtained for the same geometry. They found that all models used predicted practically satisfactory and acceptable within the current Reynolds number range.

MHD flows with Hall and ion-slip currents have applications in the design of MHD generators, Hall accelerators, and flight magnetohydrodynamics $[5,11,12]$. The Hall effect is important since it can be applied in determining if a given material is a semiconductor or insulator, and it can measure the magnetic field and also in sensing as they are immune to water, mud, dust, and dirt and in integrated circuits as Hall effect sensors. The heat-generated effects may alter the temperature distribution and, consequently, alter the rate of particle deposition in a nuclear reactor, electronic chips, and semiconductor wafers [13]. The magnitude of thermal diffusion effect may be considerably large, and it can be utilized as such in the separation of isotopes in a mixture of gases with very light molecular weight (hydrogen and helium) and gases with 
medium molecular weight (nitrogen and air). Mahmud and Mohammad [14] studied MHD fluid flow of an incompressible electrically conducting fluid along a semi-infinite vertical porous plate under strong transverse magnetic field with rotation. The momentum and energy equations were solved by the perturbation technique. Das et al. [15] investigated the effects of slip condition on an unsteady MHD flow of a viscous incompressible electrically conducting fluid past a periodically accelerated horizontal porous plate under the influence of transverse magnetic field and Hall current. The flow was generated due to the accelerated motion of the porous plate. A unified closed form analytical solution of governing equations was obtained by the Laplace transform technique. Prasad et al. [16] investigated the MHD flow and heat transfer of an electrically conducting fluid over a stretching sheet with variable thickness. The effects of external magnetic field along the sheet and the Hall currents were considered too. The governing equations were solved numerically using an implicit finite difference scheme. The results showed the effect of the Hall current which gave rise to a cross flow. Moreover, the Hall current and the magnetic field had a strong effect on the flow and heat transfer characteristics. Ram [17] employed the finite difference method to analyse the MHD Stokes problem for a vertical plate with Hall and ion-slip currents and discussed MHD Stokes problem of a convective flow of a vertical infinite plate in a dissipative rotating fluid with Hall current for the effects of various parameters.

Free convection in channel flow has many important applications in designing ventilating and heating of buildings, cooling of electronic components of a nuclear reactor, bed thermal storage, and heat sink in the turbine blades. Convective flows driven by temperature difference of the bounding walls of channels are important in industrial applications [18, 19]. Soundalgekar [3] studied magnetohydrodynamic free convection flow past an infinite vertical plate oscillating in its own plane. An isothermal plate was considered, and it was observed that a phase angle of $\omega=3 \pi / 2$ brings about cooling of the plate making the flow unstable. Bhaskar et al. [20] studied the effects of Hall currents and radiation on MHD free convection of a viscous incompressible fluid confined between two vertical walls in a rotating medium. The governing equations were solved analytically using the Laplace transform technique. Mahendar and Rao [21] investigated the effect of Hall current on an unsteady MHD free convective flow along a vertical porous plate with thermal radiation. The dimensionless governing coupled, nonlinear boundary layer partial differential equations were solved by an efficient finite element method for velocity, temperature, concentration field, and also the expression for shearing stress, Nusselt number, and Sherwood number. MHD free convective heat and mass transfers of a heat generating fluid past an impulsively started infinite vertical porous plate with Hall current and radiation absorption was studied in [22]. The effects of Hall currents on MHD free convective flow past a semi-infinite vertical plate with mass transfer for different parameters was studied in [23].

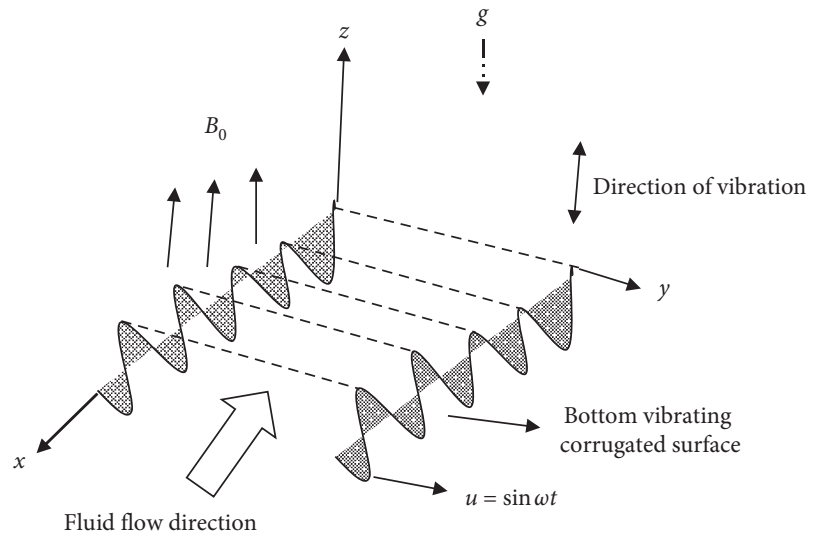

FIGURE 1: Schematic diagram with boundary.

Although different authors studied heat and mass transfers on different rotating geometric structures with or without viscous dissipation and porosity applying different numerical techniques, this study concentrates on corrugated vibrating surface with hall currents and heat and mass transfers that is sinusoidal in nature and nonporous bottom by applying the FDM explicit scheme because of stability.

\section{Mathematical Formulation}

Consider an unsteady MHD flow of an electrically conducting incompressible fluid on a vibrating bottom surface that is sinusoidal as shown in Figure 1. The $x$-axis is taken along the infinite surface and the $y$-axis normal to it. Initially the corrugated surface and the fluid are at the same temperature, and fluid is allowed to flow in the upward direction. At time $t>0$, the plate starts vibrating with a frequency of oscillation $\omega$ and reference velocity $U_{R}$ forming boundary velocity $u=U_{R} \sin \omega t$, where $U_{R}$ is the reference velocity having a linear relation with the vibration amplitude and the vibration frequency. A magnetic field $B_{0}$ of uniform strength is applied perpendicular to the plate along the positive $z$-axis. The gravitational acceleration $g$ is acting downwards.

The magnetic Reynolds number is assumed to be small since the electric intensity $\mathbf{E}$ is zero at the plate; therefore, it is assumed to be zero everywhere within the flow [24]. Considering the equation of conservation of electric charge, [25] given as $\nabla \cdot \mathbf{J}=0$, the components of electric current density along the $x, y$, and $z$-axes are $J_{\mathrm{x}}$, $J_{y}$, and $J_{z}$, respectively. Since the bottom vibrating surface is electrically nonconducting, $\mathbf{E}=0$ and $J_{z}$ is zero everywhere in the flow [26]. Therefore, considering Hall current in the problem, the generalized Ohm's law [25] is given as

$$
\mathbf{J}+\frac{\omega_{e} \tau_{e}}{\mathbf{H}_{0}} \mathbf{J} \times \mathbf{H}=\sigma\left(\mathbf{E}+\mu_{e} \mathbf{q} \times \mathbf{H}\right) .
$$

For the electron pressure gradient for weakly ionized fluid in equation (1), ion-slip and thermoelectric effect are neglected. Taking these assumptions into consideration and in the absence of electric field, it simplifies to 


$$
\begin{aligned}
& J_{x}+m J_{y}=\sigma \mu_{e} H_{o} v, \\
& J_{y}-m J_{x}=-\sigma \mu_{e} H_{o} u,
\end{aligned}
$$

where $m=\omega_{e} \tau_{e}$ is the Hall parameter. By solving equations (2) and (3) for $J_{x}$ and $J_{y}$, it gives

Applying Lorentz force $\mathbf{J} \times \mathbf{B}$ yields

$$
J_{y} B_{o} \mathbf{i}-J_{x} B_{o} \mathbf{j}
$$

If there is an appreciable temperature difference between the vibrating surface and the ambient fluid, the temperature- dependent heat source or sink is considered which may exert a strong influence on the heat transfer characteristics within the fluid [27]. Moreover, if $Q$ is the temperature-dependent volumetric heat generation parameter, then $Q>0$ represents a heat source, and $Q<0$ represents a heat sink. Incorporating equations (2) to (4) into the equations of momentum, the following respective equations of momentum, energy, and concentration are obtained:

Equations of momentum:

$$
\begin{aligned}
& \frac{\partial u}{\partial t}+u \frac{\partial u}{\partial x}+v \frac{\partial u}{\partial y}=v\left(\frac{\partial^{2} u}{\partial x^{2}}+\frac{\partial^{2} u}{\partial y^{2}}\right)+g \beta_{T}\left(T-T_{\infty}\right)+g \beta_{C}\left(C-C_{\infty}\right)-\frac{\sigma \mathrm{B}_{0}^{2}}{\rho\left(1+m^{2}\right)}(m v-u) \\
& \frac{\partial v}{\partial t}+u \frac{\partial v}{\partial x}+v \frac{\partial v}{\partial y}=v\left(\frac{\partial^{2} v}{\partial x^{2}}+\frac{\partial^{2} v}{\partial y^{2}}\right)-\frac{\sigma \mathrm{B}_{0}^{2}}{\rho\left(1+m^{2}\right)}(v+m u) .
\end{aligned}
$$

Energy equation:

$$
\begin{aligned}
& \frac{\partial T}{\partial t}+u \frac{\partial T}{\partial x}+v \frac{\partial T}{\partial y} \\
& =\frac{\kappa}{\rho \mathbf{C}_{p}}\left(\frac{\partial^{2} T}{\partial x^{2}}+\frac{\partial^{2} T}{\partial y^{2}}\right)+\frac{\mu}{\rho \mathbf{C}_{p}}\left[\left(\frac{\partial u}{\partial x}\right)^{2}\right. \\
& \left.+\left(\frac{\partial v}{\partial x}\right)^{2}\right]+\frac{D_{M} k_{T}}{\mathbf{C}_{p \mathbf{C}_{s}}}\left(\frac{\partial^{2} C}{\partial x^{2}}+\frac{\partial^{2} C}{\partial y^{2}}\right)+\frac{\sigma B_{0}^{2}}{\rho \mathbf{C}_{p}}\left(u^{2}+v^{2}\right) \\
& +\frac{Q_{0}}{\rho \mathbf{C}_{p}}\left(T-T_{\infty}\right)-\frac{16 \sigma_{s} T_{\infty}^{3}}{3 \rho \mathbf{C}_{p} k_{e}} \frac{\partial^{2} T}{\partial x^{2}} .
\end{aligned}
$$

Equation of concentration:

$$
\frac{\partial C}{\partial t}=D_{M}\left(\frac{\partial^{2} C}{\partial x^{2}}+\frac{\partial^{2} C}{\partial y^{2}}\right)+\frac{D_{T} k_{T}}{T_{m}}\left(\frac{\partial^{2} T}{\partial x^{2}}+\frac{\partial^{2} T}{\partial y^{2}}\right)-u \frac{\partial C}{\partial x}-v \frac{\partial C}{\partial y}
$$

The initial and boundary conditions for the current problem are specified as follows:

$$
\begin{array}{lll}
t=0: & u=0, v=0, T=0, C=0, \quad \text { at } 0 \leq x \leq L, \\
t>0: & u=U_{R} \sin \omega t, U=U_{\infty}, C=C_{w}, T=T_{w}, C=C_{\infty}, \quad \text { at } x=0, \\
& T=T_{\infty}+\left(T_{w}-T_{\infty}\right), v=0, C=C_{\infty}+\left(C_{w}-C_{\infty}\right), & \text { at } y=0 \\
& u=0, v=0, T=T_{\infty}, C=C_{\infty}, \quad \text { at } x=L . &
\end{array}
$$

\section{Nondimensionalization}

The nondimensional equations of momentum, energy, and concentration are transforming the equations governing the flow to their respective nondimensionalised form given as

$$
\begin{aligned}
& \frac{\partial u^{*}}{\partial t^{*}}+u^{*} \frac{\partial u^{*}}{\partial x^{*}}+v^{*} \frac{\partial u^{*}}{\partial y^{*}}=\frac{1}{R_{e}}\left(\frac{\partial^{2} u^{*}}{\partial x^{* 2}}+\frac{\partial^{2} u^{*}}{\partial y^{* 2}}\right)+\frac{M}{1+m^{2}}\left(m v^{*}-u^{*}\right)+G r_{\theta} T^{*}+G r_{m} C^{*}-M u^{*}, \\
& \frac{\partial v^{*}}{\partial t^{*}}+u^{*} \frac{\partial v^{*}}{\partial x^{*}}+v^{*} \frac{\partial v^{*}}{\partial y^{*}}=\frac{1}{R_{e}}\left(\frac{\partial^{2} v^{*}}{\partial x^{* 2}}+\frac{\partial^{2} v^{*}}{\partial y^{* 2}}\right)+\frac{M}{1+m^{2}}\left(v^{*}+m u^{*}\right)-M u^{*},
\end{aligned}
$$




$$
\begin{aligned}
\frac{\partial T^{*}}{\partial t^{*}}+u^{*} \frac{\partial T^{*}}{\partial x^{*}}+v^{*} \frac{\partial T^{*}}{\partial y^{*}}= & \frac{1}{R_{e}}\left[\frac{1}{P_{r}}\left(\frac{\partial^{2} T^{*}}{\partial x^{* 2}}+\frac{\partial^{2} T}{\partial y^{* 2}}\right)\right]+\frac{E c}{R_{e}}\left[\left(\frac{\partial u^{*}}{\partial x^{*}}\right)^{2}+\left(\frac{\partial v^{*}}{\partial x^{*}}\right)^{2}\right]+Q T^{*}+D_{f} R_{e}\left(\frac{\partial^{2} C^{*}}{\partial x^{* 2}}+\frac{\partial^{2} C^{*}}{\partial y^{* 2}}\right) \\
& +R R_{e}\left(u^{* 2}+v^{* 2}\right)-\left(\frac{4}{3 N P_{r} R_{e}} \frac{\partial^{2} T^{*}}{\partial x^{* 2}}\right)
\end{aligned}
$$

where $Q=Q_{0} H / \rho U C_{p}$, the nondimensional heat, is the source or sink parameter [28].

$$
\begin{aligned}
\frac{\partial C^{*}}{\partial t^{*}}+u^{*} \frac{\partial C^{*}}{\partial x^{*}}+v^{*} \frac{\partial C^{*}}{\partial y^{*}}= & \frac{1}{S c R_{e}}\left(\frac{\partial^{2} C^{*}}{\partial x^{* 2}}+\frac{\partial^{2} C^{*}}{\partial y^{* 2}}\right) \\
& +\frac{S_{r}}{R_{e}}\left(\frac{\partial^{2} T^{*}}{\partial x^{* 2}}+\frac{\partial^{2} T^{*}}{\partial y^{* 2}}\right) .
\end{aligned}
$$

The nondimensional form of the initial and boundary conditions for this problem is given as

$$
\begin{array}{ll}
t^{*}=0: & u^{*}=0, v^{*}=0, T^{*}=0, C^{*}=0, \quad \text { at } 0 \leq x^{*} \leq L, \\
t^{*}>0: & u^{*}=\sin \omega t, v^{*}=0, T^{*}=1, C^{*}=1, \quad \text { at } x^{*}=0, \\
& u^{*}=0, v^{*}=0, T^{*}=T_{\infty}+\left(T_{w}-T_{\infty}\right), C^{*}=C_{\infty}+\left(C_{w}-C_{\infty}\right), \quad \text { at } y^{*}=0, \\
& u^{*}=0, v^{*}=0, T^{*}=1, C^{*}=1, \quad \text { at } x^{*}=L .
\end{array}
$$

The equations that govern the flow on a vibrating corrugated surface in the presence of heat source or sink are coupled and highly nonlinear. They are represented by equations (10)-(12) and are to be solved under the initial and boundary conditions (13).

\section{Numerical Technique}

The numerical method used to solve the equations governing the flow together with the initial and boundary conditions applies the forward-time backward-space (FTBS) difference method which is stable and convergent. A necessary condition for time stability is the CFL condition which depends on time and space discretization.

The finite difference form of the equations of momentum (14) and (15), concentration (16), and energy) (17) are given as

$$
\begin{aligned}
U_{i, j}^{k+1}= & {\left[U_{i, j}^{k}-\frac{\Delta t}{2 \Delta x} U_{i, j}^{k}\left(-U_{i-1, j}^{k+1}+U_{i, j}^{k}-U_{i-1, j}^{k}\right)+\frac{\Delta t}{2 R_{e}(\Delta x)^{2}}\left(U_{i+1, j}^{k+1}+U_{i-1, j}^{k+1}+U_{i+1, j}^{k}-2 U_{i, j}^{k}+U_{i-1, j}^{k}\right)+\frac{\Delta t}{2 R_{e}(\Delta y)^{2}}\right.} \\
& \cdot\left(U_{i, j+1}^{k+1}+U_{i, j-1}^{k+1}+U_{i, j+1}^{k}-2 U_{i, j}^{k}+U_{i, j-1}^{k}\right)+\frac{M \Delta t}{2+2 m^{2}}\left(m\left(V_{i, j}^{k+1}+V_{i, j}^{k}\right)-U_{i, j}^{k}\right)-M \frac{\Delta t}{2} U_{i, j}^{k}+\frac{\Delta t G r_{\theta}}{2}\left(T_{i, j}^{k+1}+T_{i, j}^{k}\right) \\
+ & \left.\frac{\Delta t G r_{m}}{2}\left(C_{i, j}^{k+1}+C_{i, j}^{k}\right)\right] \cdot\left[1+U_{i, j}^{k} \frac{\Delta t}{2 \Delta x}+\frac{\Delta t}{R_{e}(\Delta x)^{2}}+\frac{\Delta t}{R_{e}(\Delta y)^{2}}+\frac{1}{2} M \Delta t+\frac{M \Delta t}{2+2 m^{2}}\right]^{-1}, \\
V_{i, j}^{k+1}= & {\left[V_{i, j}^{k}-\frac{\Delta t}{2 \Delta x} V_{i, j}^{k}\left(-V_{i-1, j}^{k+1}+V_{i, j}^{k}-V_{i-1, j}^{k}\right)+\frac{\Delta t}{2 R_{e}(\Delta x)^{2}}\left(V_{i+1, j}^{k+1}+V_{i-1, j}^{k+1}+V_{i+1, j}^{k}-2 V_{i, j}^{k}+V_{i-1, j}^{k}\right)\right.} \\
& \left.+\frac{\Delta t}{2 R_{e}(\Delta y)^{2}}\left(V_{i, j+1}^{k+1}+V_{i, j-1}^{k+1}+V_{i, j+1}^{k}-2 V_{i, j}^{k}+V_{i, j-1}^{k}\right)+\frac{M \Delta t}{2+2 m^{2}}\left(V_{i, j}^{k}+m\left(U_{i, j}^{k+1}-U_{i, j}^{k}\right)\right)-M \frac{\Delta t}{2} V_{i, j}^{k}\right] \\
& \cdot\left[1+U_{i, j}^{k} \frac{\Delta t}{2 \Delta x}+\frac{\Delta t}{R_{e}(\Delta x)^{2}}+\frac{\Delta t}{R_{e}(\Delta y)^{2}}+\frac{1}{2} M \Delta t-M \frac{\Delta t}{2+2 m^{2}}\right]^{-1},
\end{aligned}
$$




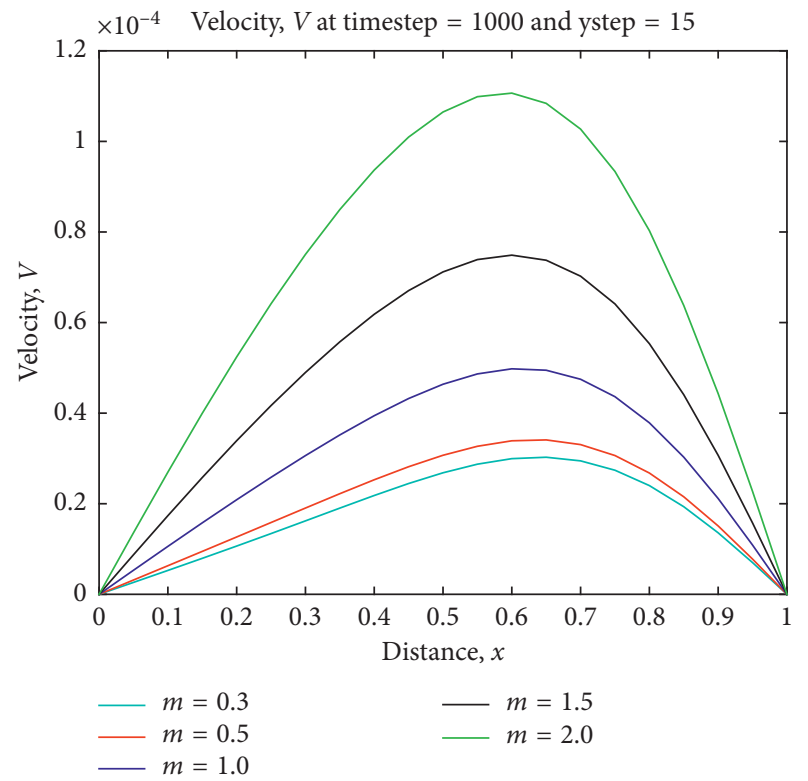

FIGURE 2: Graph of effects of Hall parameters on secondary velocity profile $v$.

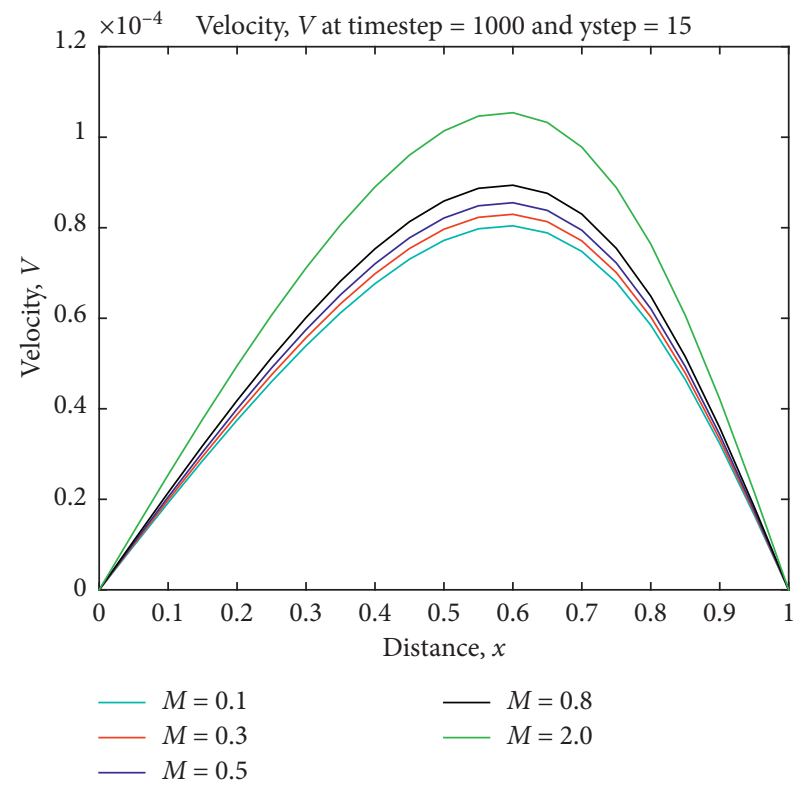

FIGURE 3: Graph of effects of the magnetic parameter on secondary velocity profile $v$.

$$
\begin{aligned}
C_{i, j}^{k+1}= & {\left[C_{i, j}^{k}-\frac{\Delta t}{2 \Delta x} U_{i, j}^{k}\left(-C_{i-1, j}^{k+1}+C_{i, j}^{k}-C_{i-1, j}^{k}\right)+\frac{\Delta t}{2 R_{e} s c(\Delta x)^{2}}\left(C_{i+1, j}^{k+1}+C_{i-1, j}^{k+1}+C_{i+1, j}^{k}-2 C_{i, j}^{k}+C_{i-1, j}^{k}\right)\right.} \\
& +\frac{\Delta t}{2 R_{e} s c(\Delta y)^{2}}\left(C_{i, j+1}^{k+1}+C_{i, j-1}^{k+1}+C_{i, j+1}^{k}-2 C_{i, j}^{k}+C_{i, j-1}^{k}\right)+\frac{S_{r} \Delta T}{2 R_{e}(\Delta x)^{2}}\left(T_{i+1, j}^{k+1}+T_{i-1, j}^{k+1}+T_{i+1, j}^{k}-2 T_{i, j}^{k}+T_{i-1, j}^{k}\right) \\
& \left.-\frac{S_{r} \Delta T}{2 R_{e}(\Delta y)^{2}}\left(T_{i, j+1}^{k+1}+T_{i, j-1}^{k+1}+T_{i, j+1}^{k}-2 T_{i, j}^{k}+T_{i, j-1}^{k}\right)\right] \cdot\left[1+\frac{\Delta t}{2 \Delta x} U_{i, j}^{k}+\frac{\Delta t}{2 R_{e} s c(\Delta x)^{2}}+\frac{\Delta t}{2 R_{e} s c(\Delta y)^{2}}\right]^{-1}, \\
T_{i, j}^{k+1}= & {\left[T_{i, j}^{k}-\frac{Q \Delta t}{2} T_{i, j}^{k}-\frac{\Delta t}{2 \Delta x} U_{i, j}^{k}\left(-T_{i-1, j}^{k+1}+T_{i, j}^{k}-T_{i-1, j}^{k}\right)+\frac{D_{f} \Delta t}{2 R_{e}(\Delta x)^{2}}\left(C_{i+1, j}^{k+1}+C_{i-1, j}^{k+1}+C_{i+1, j}^{k}-2 C_{i, j}^{k}+C_{i-1, j}^{k}\right)\right.} \\
+ & \frac{D_{f} \Delta t}{2 R_{e}(\Delta y)^{2}}\left(C_{i, j+1}^{k+1}+C_{i, j-1}^{k+1}+C_{i, j+1}^{k}-2 C_{i, j}^{k}+C_{i, j-1}^{k}\right)+\frac{E_{c} \Delta t}{4 R_{e}(\Delta x)^{2}}\left(U_{i, j}^{k+1}+U_{i-1, j}^{k+1}+U_{i, j}^{k}-U_{i-1, j}^{k}\right)^{2} \\
+ & \left.\frac{E_{c} \Delta t}{4 R_{e}(\Delta y)^{2}}\left(U_{i, j}^{k+1}+U_{i, j-1}^{k+1}+U_{i, j}^{k}-U_{i, j-1}^{k}\right)^{2}\right] \cdot\left[1+U_{i, j}^{k}+\frac{\Delta t}{2 R_{e} \operatorname{Pr}(\Delta x)^{2}}\left(T_{i+1, j}^{k+1}+T_{i-1, j}^{k+1}+T_{i+1, j}^{k}-2 T_{i, j}^{k}+T_{i-1, j}^{k}\right)\right. \\
+ & \frac{\Delta t}{2 R_{e} \operatorname{Pr}(\Delta y)^{2}}\left(T_{i, j+1}^{k+1}+T_{i, j-1}^{k+1}+T_{i, j+1}^{k}-2 T_{i, j}^{k}+T_{i, j-1}^{k}\right)+\frac{\Delta t R R_{e}}{4}\left(\left(U_{i, j}^{k}\right)^{2}+\left(V_{i, j}^{k}\right)^{2}\right)-\frac{2 \Delta t}{3 N R_{e} \operatorname{Pr}(\Delta y)^{2}} \\
+ & \left.\left(T_{i, j+1}^{k+1}+T_{i, j-1}^{k+1}+T_{i, j+1}^{k}-2 T_{i, j}^{k}+T_{i, j-1}^{k}\right)+\frac{\Delta t}{2 \Delta x}+\frac{\Delta t}{R_{e} \operatorname{Pr}(\Delta x)^{2}}+\frac{\Delta t}{R_{e} \operatorname{Pr}(\Delta y)^{2}}-\frac{Q^{2} \Delta t}{2}-\frac{4 \Delta t}{3 N R_{e} \operatorname{Pr}(\Delta y)^{2}}\right]^{-1} .
\end{aligned}
$$

A mesh size is fixed at $\Delta x=0.2, \Delta y=0.3$, and $\Delta t=0.0001$, where a sinusoidal shape is formed by the $x$-axis and $y$-axis considered appropriate for calculation of velocities, temperature, and concentration. The FTBS scheme is unconditionally stable, and thus compatibility and stability ensures convergence. Hence, the present employed scheme is always convergent (Figure 2).

\section{Discussion of the Results}

In order to ascertain the accuracy of the numerical results, the present study is compared with the available solution in literature. The velocity, temperature, and concentration fields have been discussed by assigning numerical values to various nondimensional parameters, that is $G r_{\theta}=10, G r_{m}=10, M=0.2, E c=0.5, D_{f}=0.03$, $S r=2.0, S c=0.22, R=0.2, \omega t=30, m=0.5$, and $Q=0.8$. The values of the Schmidt number used are $0.22,0.62$, and 0.78 and represent hydrogen, water vapour, and ammonia, respectively, at $20^{\circ} \mathrm{C}$ and one atmosphere pressure. It is clear from Figures 1 and 3 that, in the presence of a heat source, the increase in Hall parameter $m$ leads to an increase in the magnitude of primary and secondary velocity profiles. The Hall parameter $m$ 
increases the transverse velocity profile up to a value $m=1.5$; however, for the values $m>1.5$, the transverse velocity profiles decreases since the large values of $m$ turn $M /\left(m+m^{2}\right)$ very small, and hence the resistive effect of magnetic field diminishes. This is due to the fact that an increase in $m$ decreases the effective conductivity and hence the magnetic damping.

An increase in the magnetic parameter $M$ leads to a decrease in the magnitude of primary velocity profiles and secondary velocity profiles, respectively. A large value of $M$ causes a reversal in the direction of the secondary velocity profiles. The effect of $M$ results to a decreasing velocity distribution across the boundary layer due to the effect of transverse magnetic field which gives rise to Lorentz force that has a tendency to flow down the fluid motion. A higher $M$ increases the transverse velocity field and reaches a peak value near the vicinity of the boundary layer and approaches zero, and the less magnetic field has less peak value as it reaches zero far away from the boundary.

It is clear from Figure 4 that, for lower values of $S c$, it decreases the concentration distribution in the boundary layer thickness, which is associated with the reduction in the concentration profiles. The particles have greater diffusivity conditioned by viscosity of the medium for them to cross from laminar to turbulent-characterized fluid flows in which there are simultaneous momentum and mass diffusion convection processes. This means that an increase in $S c$ means a decrease of molecular diffusion; hence, the concentration of the species is higher for large values of $S c$ and lower for small values of Sc. It is noticed that the effect of increasing values of $S c$ results in a decreasing concentration profiles across the boundary layer. Physically, the rise in the value of Schmidt number means reduction of molecular diffusion. Hence, the concentration of the species is advanced for smaller values of Sc and lesser for larger values of Sc.

It is observed from Figure 5 that an increase in the Prandtl number decreases the velocity of the flow field. Since Prandtl number is the ratio of kinematic viscosity to thermal diffusivity, this implies that an increase in the kinematic viscosity of the fluid dominates the thermal diffusivity of the fluid which leads to decreasing the velocity of the flow field.

Figure 6 presents the effect of the Prandtl number Pr on the temperature profiles as a result of the heat source $(Q>0)$. An increase in the value of Pr has the tendency to decrease the fluid temperature in the boundary layer as well as the thermal boundary layer thickness. This causes the wall slope of the temperature to decrease as Pr increases. This means that a mixture of flue gas $(S c=0.64)$ and hydrogen $(S c=0.22)$ diffuses faster than does a mixture of air and hydrogen; and a mixture of air and hydrogen diffuses faster than a mixture of water and hydrogen. Figure 5 shows that, in the presence of a heat source, an increase in the Schmidt number $S c$ causes a decrease in the velocity profiles.

A similar condition is experienced as shown in Figure 7, where an increase Pr over a given time causes a decrease in concentration profile.

Figures 8 and 9 show that an increase in the magnitude of the heat sink parameter $(Q<0)$ leads to a decrease in the

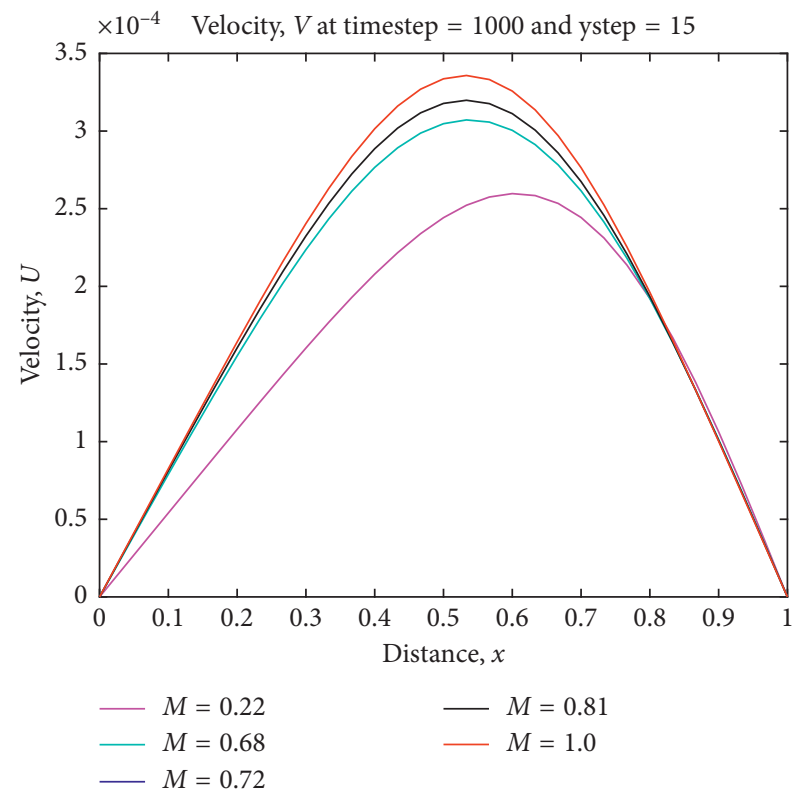

FIgURE 4: Graph of effects of the Schmidt number on primary velocity profile $u$.

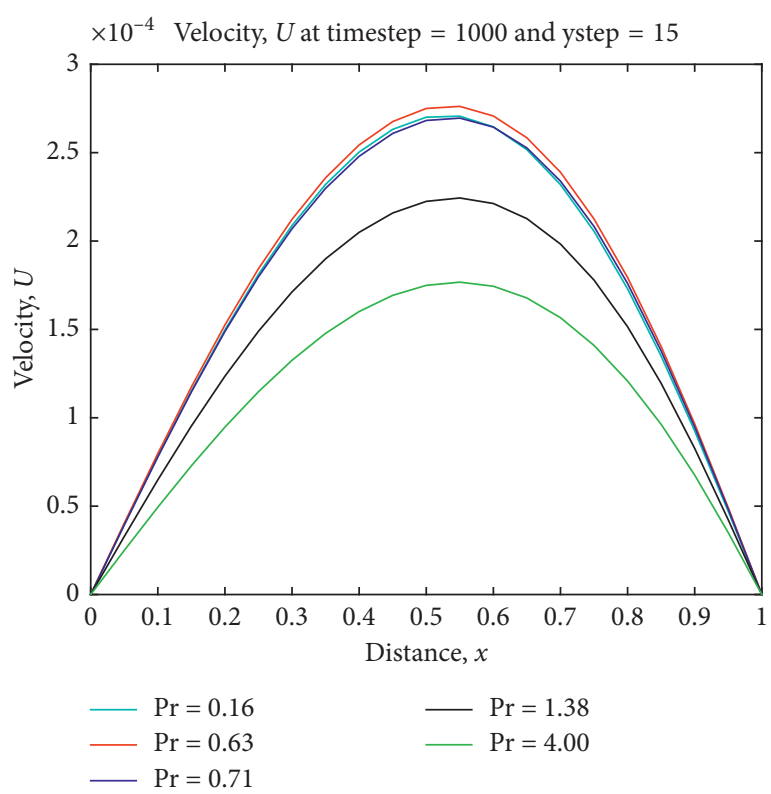

FIgURe 5: Graph of effects of the Prandt number on primary velocity profile $u$.

magnitude of temperature and velocity profiles, respectively. A contrary effect is shown in Figure 8, where an increase in $Q$ leads to an increase in concentration profiles since the presence of a heat sink produces a cooling effect that decreases velocity of the convection currents that move upwards next to the surface of the vibrating surface, leading to higher concentration profiles.

Heat source is an indication that generation of heat from the surface (this is due to $T_{w}>T_{\infty}$ ), which increases the temperature in the flow field. This physically implies that when the heat source parameter increased, the temperature 


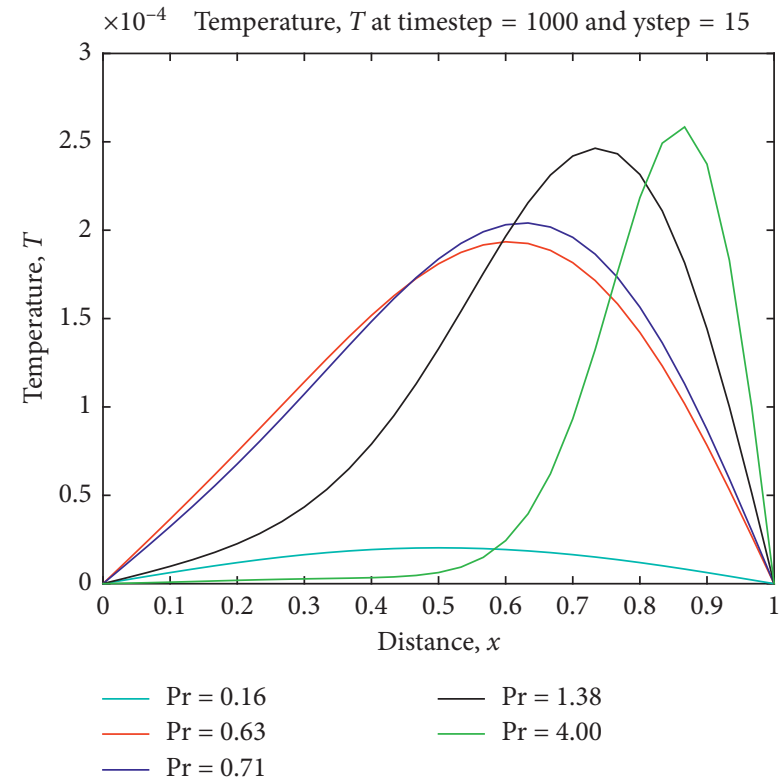

Figure 6: Graph of effects of the Prandt number on temperature profile $T$.

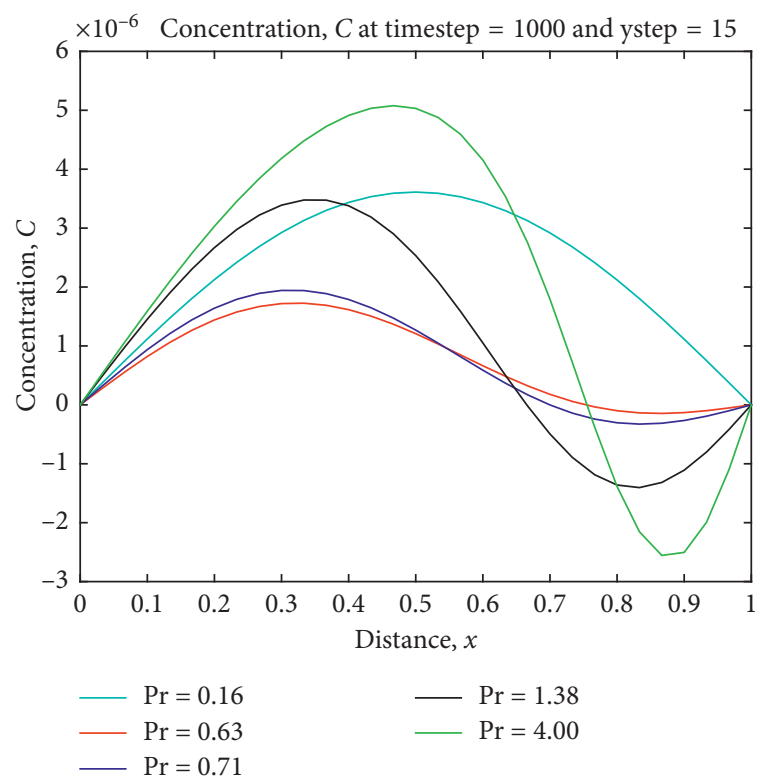

Figure 7: Graph of effects of the Prandt number on concentration profile $C$.

increases steeply and exponentially from the surface. The influence of heat source parameter $Q>0$ on velocity and temperature profiles is very much significantly related to the heat sink parameter $Q<0$ (Figure 10).

As Re changes, separation of flows in the weak region of the corrugated vortices is created. At lower Re, no separation occurs, and an increase in Re unstable separations are seen as a result of vortex shedding at certain frequencies, and it applies that the viscous force is predominant, thus imposing drug in the fluid and thus

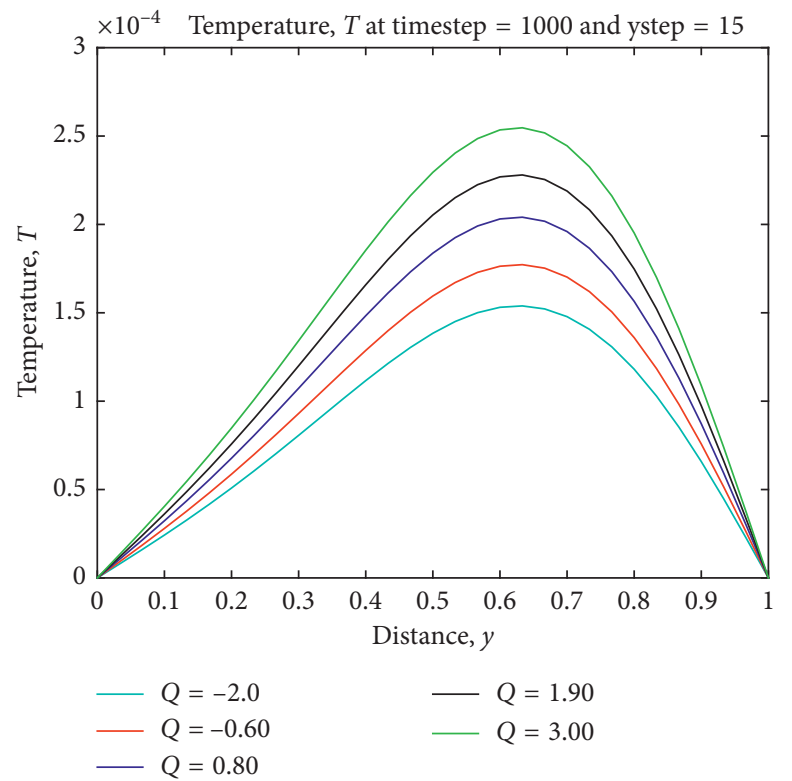

FIGURE 8: Graph of effects of heat source/sink parameter on temperature profile $T$.

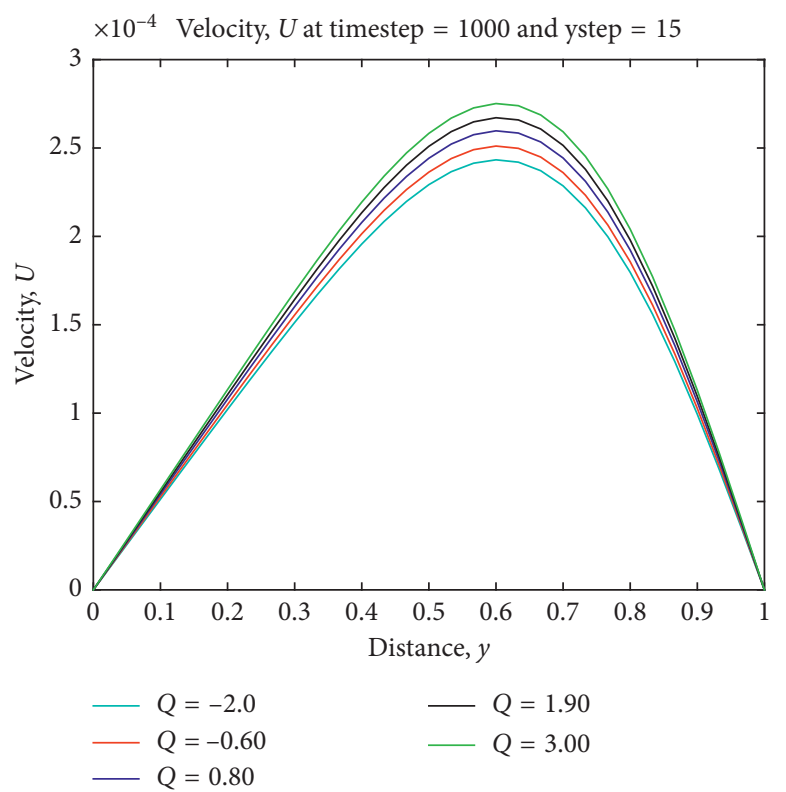

FIGURE 9: Graph of effects of heats source/sink parameter on primary velocity profile $u$.

decreasing the fluid flow as shown in Figure 11. The effect of the flow velocity on the rod vibration can be represented by the Reynolds number, which expresses the extent of the flow turbulence.

The Nusselt number physically indicates the rate of heat transfer and the local Sherwood number as well as the rate of mass transfer. The shearing stress on the surface of the vibrating surface is defined. The local skin friction coefficients due to the primary and the secondary velocity profiles are given as 


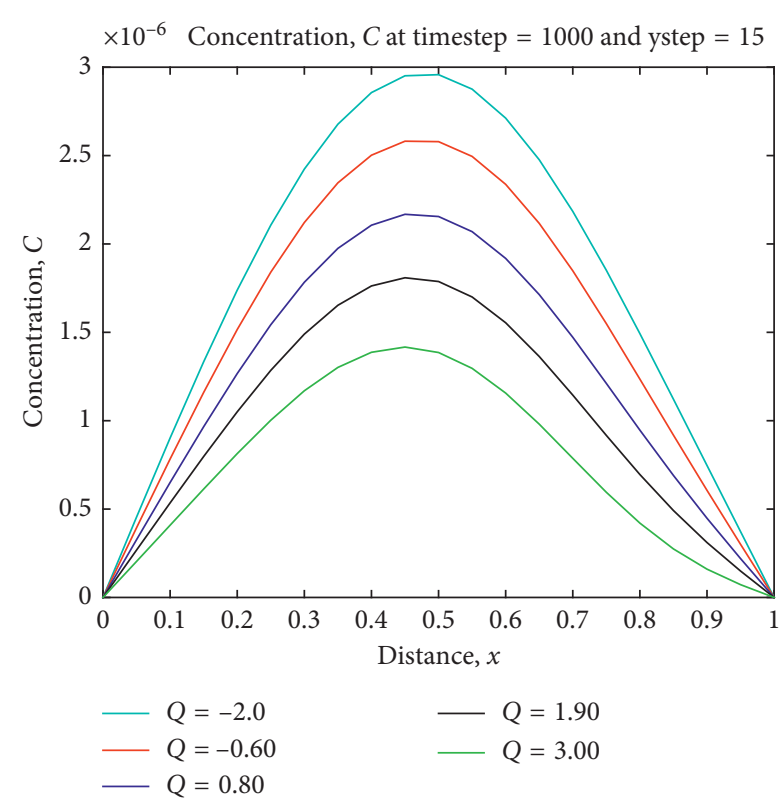

Figure 10: Graph of effects of heat source/sink parameter on concentration profile $C$.

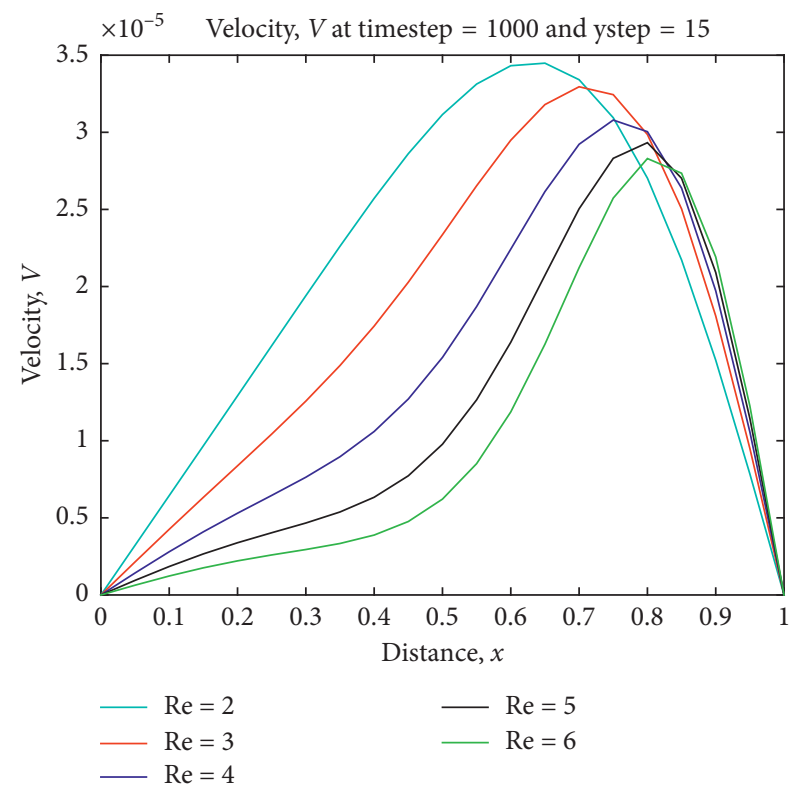

FIGURE 11: Graph of effects of the Reynolds number on secondary velocity profile.

$$
\begin{aligned}
& C_{f x}=-\frac{2}{\operatorname{Re} U^{2}}\left(\frac{\partial u}{\partial x}\right)_{x=0}, \\
& C_{f y}=-\frac{2}{\operatorname{Re} U^{2}}\left(\frac{\partial v}{\partial x}\right)_{x=0} .
\end{aligned}
$$

The length $L$ units along a vibrating surface is expressed in Sherwood number as

$$
S h=-\left(\frac{\partial C}{\partial x}\right)_{x=0} .
$$

TABLE 1: Values of the Nusselt number, Sherwood number, and skin friction.

\begin{tabular}{lcccc}
\hline Parameters & $\mathbf{C}_{f x}$ & $\mathbf{C}_{f y}$ & $N u$ & $S h$ \\
\hline $\operatorname{Pr}=0.71$ & -0.3114 & 0.00291 & 0.06961 & 1.19124 \\
$\operatorname{Pr}=0.63$ & -0.03132 & 0.00284 & 0.03997 & 1.20154 \\
$N=3$ & -0.05287 & 0.00841 & 0.71812 & 1.87443 \\
$m=1.5$ & -0.02993 & 0.00106 & 0.0108 & 1.15884 \\
$m=0.5$ & -0.02874 & 0.00229 & 0.06942 & 1.19442 \\
$M=0.1$ & -0.01974 & 0.00267 & 0.0681 & 1.20014 \\
$M=2.0$ & -0.01971 & 0.00183 & 0.15717 & 1.19331 \\
$Q=0.80$ & -0.02768 & 0.002513 & 0.06800 & 1.18772 \\
$Q=1.90$ & -0.03001 & 0.00291 & 0.02681 & 1.20013 \\
\hline
\end{tabular}

Furthermore, the local Nusselt number $\mathrm{Nu}$ may be expressed as

$$
N_{u}=-\left(\frac{\partial T}{\partial x}\right)_{x=0}
$$

Heat and mass transfers are considered. This is done by computing velocity, concentration, and temperature in equations (18) to (21) in MATLAB code to generate the values of skin friction, Nusselt number, and Sherwood number as shown in Table 1.

It is clear from the table that

(i) As time increases, a decrease in Nusselt number, Sherwood number, and Skin friction occurs as it physically implies that shear stresses decrease with an increase in time.

(ii) An increase in the heat source parameter leads to an increase in both skin frictions, meaning that an increase in $Q$ enhances convection currents on the flow and a reverse effect in Sherwood number and Nusselt number since a thicker thermal boundary layer leading to lower temperature gradients.

(iii) A high value of radiation parameter leads to an increase in magnitudes of skin frictions and Nusselt number as a result of an increase in the rate of species concentration. It is clear that the effect of radiation is to decrease the rate of energy transport to the fluid, thereby decreasing the temperature of the fluid but decreasing the Sherwood number.

\section{Conclusion}

A numerical study has been conducted on free convective heat and mass transfers of an incompressible electrically conducting fluid on a vibrating bottom surface with Hall currents and heat and mass transfers. It is clear that

(i) The velocity profiles decreases with an increase in the Prandtl number Pr, Schmidt number Sc, Magnetic parameter $M$, and Soret number $S r$.

(ii) Concentration profiles increase with an increase in the Reynolds number Re and decreases with an increase in the Prandtl number Pr and Schmidt number Sc. 
(iii) Both hydrodynamic and thermal behaviours are relevant to the Reynolds number, and the patterns are shown by oscillations.

(iv) Fluid damping is generated as the bottom surface vibrates normal to the flow, and this is proportional to the surface velocity. Therefore, an increase in damping causes an increase in the velocity.

(v) It is shown that an increase in the parameters under consideration causes reduction in the skin friction.

\section{Nomenclature}

$B_{0}$ : $\quad$ Magnetic field strength, $\mathrm{Wbm}^{-1}$

B: $\quad$ Magnetic flux density, $\mathrm{Wbm}^{-2}$

Sc: $\quad$ Schmidt number

$S_{r}: \quad$ Soret number

$D_{f}: \quad$ Dufour number

$D_{M}:$ Molecular diffusion coefficient

M: $\quad$ Magnetic parameter

$T: \quad$ Dimensional temperature of the fluid, $\mathrm{K}$

$R: \quad$ Joules heating parameter

$N$ : Radiative parameter

g: $\quad$ Acceleration due to gravity $\left(\mathrm{m} \cdot \mathrm{s}^{-2}\right)$

Pr: Prandtl number

Ec: $\quad$ The Eckert number

D: $\quad$ Electric flux density, $\left(\mathrm{C} \cdot \mathrm{m}^{-2}\right)$

H: $\quad$ Magnetic field intensity vector, $\left(\mathrm{A} \cdot \mathrm{m}^{-1}\right)$

$L: \quad$ Characteristic length $(\mathrm{m})$

$\omega t$ : Phase angle

$G r_{\theta}: \quad$ Local temperature Grashof number

$\mathrm{Gr}_{m}$ : Local mass Grashof number

$\omega: \quad$ Frequency of oscillation

$T_{\infty}: \quad$ Fluid temperature far from the surface of the object) $(\mathrm{K})$

$T_{s}: \quad$ Wall surface temperature $(\mathrm{K})$

$C_{\infty}$ : Concentration in the fluid away from the surface

$R_{e}: \quad$ Hydromagnetic Reynold's number

E: $\quad$ Electric field strength, $\mathrm{V} \cdot \mathrm{m}^{-1}$

M: $\quad$ Magnetic parameter

$T: \quad$ Dimensional temperature of the fluid, $\mathrm{K}$

g: $\quad$ Acceleration due to gravity $\left(\mathrm{m} \cdot \mathrm{s}^{-2}\right)$

$u, v:$ Dimensionless velocity components

$x, y$ : Cartesian coordinates

$u^{*}: \quad$ Nondimensional velocity components in $x^{*}$ and $y^{*}$

$v^{*}: \quad$ directions.

Q: Dimensionless heat source parameter

$Q_{0}: \quad$ Volumetric heat generating parameter $\left(\mathrm{W} \cdot \mathrm{m}^{-3}\right)$

$C_{s}$ : $\quad$ Concentration susceptibility parameter $\left(\mathrm{kmol} \cdot \mathrm{m}^{-3}\right)$

$C_{p}: \quad$ Specific heat at constant pressure $\left(\mathrm{J} \cdot \mathrm{Kg}^{-1} \cdot \mathrm{K}^{-1}\right)$

m: Hall parameter

$e_{e}: \quad$ Charge density, $\left(\mathrm{C} \cdot \mathrm{m}^{-3}\right)$

$C_{f x}$ : The local skin friction coefficient due the primary velocity profiles.

$C_{f y}:$ The local skin friction coefficient due the secondary velocity profiles.

$\tau_{x}$ : Viscous stress due to primary velocity profiles,

$\left(\mathrm{N} \cdot \mathrm{m}^{-2}\right.$ )

$\tau_{y}$ : Viscous stress due to secondary velocity profiles,

$\left(\mathrm{N} \cdot \mathrm{m}^{-2}\right)$

$\nabla: \quad$ Gradient operator
Greek Symbols

$\beta$ : Thermal expansion coefficient $\left(\mathrm{K}^{-1}\right)$

$\sigma$ : Electrical conductivity, $\Omega^{-1} \mathrm{~m}^{-1}$

$\rho$ : Density $\left(\mathrm{kg} \cdot \mathrm{m}^{-3}\right)$

$\omega_{e}$ : Electron frequency $\left(\mathrm{s}^{-1}\right)$

$\tau_{e}:$ Electron collision time $(t)$

$\sigma_{s}:$ Stefan-Boltzmann constant

$\mu_{e}$ : Magnetic permeability, $\left(\mathrm{H} \cdot \mathrm{m}^{-1}\right)$

$\beta_{T}$ : Thermal expansion coefficient

$\beta_{c}$ : Mass expansion coefficient

$\mu$ : Dynamic viscosity $\left(\mathrm{m}^{2} \cdot \mathrm{s}^{-1}\right)$

$v$ : Kinematic viscosity $\left(\mathrm{m}^{2} \cdot \mathrm{s}^{-1}\right)$

$\alpha$ : Thermal diffusivity $\left(\mathrm{m}^{2} \cdot \mathrm{s}^{-1}\right)$.

\section{Data Availability}

The data was generated by MATLAB version 8.5.0.197613 (R2015a) computer program.

\section{Disclosure}

This research work is an academic paper as a requirement for $\mathrm{PhD}$ completion, therefore self-sponsored by the student.

\section{Conflicts of Interest}

The authors declare that there are no conflicts of interest regarding the publication of this paper.

\section{References}

[1] Y. Perets, B. Rivin, I. Bronstein, R. Harari, and E. Sher, "Flow induced vibration of corrugated rod subjected to axial flow," WIT Transactions on the Built Environment, vol. 105, pp. 129-133, 2009.

[2] M. Amielh, F. Anselmet, Y. Jiang et al., "Aeroacoustic source analysis in a corrugated flow pipe using low-frequency mitigation," Journal of Turbulence, vol. 15, no. 10, pp. 650-676, 2014.

[3] O. Veli, T. Nehir, and S. Besir, "Flow characteristics and heat transfer enhancement in 2DCorrugated channels," International Journal of Mechanical, Aerospace, Industrial and Mechatronics Engineering, vol. 7, 2013.

[4] V. M. Soundalgekar, "Free convection effects on the flow past an infinite vertical oscillating plate," Astrophysics and Space Science, vol. 64, no. 1, pp. 165-171, 1979.

[5] R. B. Bird and W. E. S. Lightfoot, Transport Phenomena, Wiley, New York, NY, USA, 1966.

[6] V. Garg and P. Maji, "Flow and heat-transfer in a sinusoidally curved channel," International Journal of Engineering Fluid Mechanics, pp. 293-319, 1988.

[7] J. A. Gbadeyan, T. L. Oyekunle, P. F. Fasogbon, and J. U. Abubakar, "Soret and Dufour effects on heat and mass transfer in chemically reacting MHD flow through a wavy channel," Journal of Taibah University of Science, vol. 12, pp. 630-651, 2018.

[8] E. M. Abo-Eldahab and E. Aziz, "Hydrodymagnetic threedimensional flow over a stretching surface with heat and mass transfer," Heat and Mass Transfer, vol. 41, no. 8, pp. 734-743, 2005.

[9] A. S. Idowu, A. Jimoh, and L. O. Ahmed, "Impact of heat and mass transfer on MHD oscillatory flow of Jeffery fluid in a 
porous channel with thermal conductivity, Dufour and Soret," Journal of Applied Sciences Environment Management, vol. 19, no. 4, pp. 819-830, 2015.

[10] L. Zhang and D. che, "Turbulence models for fluid flow and heat transfer between cross- corrugated plates," Numerical Heat Transfer, Part A: Applications, vol. 60, no. 5, pp. 410-440, 2011.

[11] S. K. Tania and M. A. Samad, "Effects of radiation, heat generation and viscous dissipation on MHD free convection flow along a stretching sheet," Research Journal of Applied Sciences, Engineering and Technology, vol. 2, pp. 368-377, 2010.

[12] J. O’Brien and E. Sparrow, "Corrugated-duct heat transfer, pressure drop, and flow visualization," ASME Journal of Heat Transfer, vol. 104, no. 1, pp. 410-416, 1982.

[13] L. Goldstein and E. M. Sparrow, "Heat/mass transfer characteristics for flow in a corrugated wall channel," Journal of Heat Transfer, vol. 99, no. 2, pp. 187-195, 1977.

[14] A. Mahmud and R. Mohammad, "MHD fluid flow of an incompressible electrically conducting fluid along a semiinfinite vertical porous plate under strong transverse magnetic field with rotation," in Proceedings of the 8th BSME International Conference on Thermal Engineering, Dhaka, Bangladesh, December 2019.

[15] S. Das, B. Tarafdar, and R. N. Jana, "Hall effects on unsteady MHD rotating flow past a periodically accelerated porous plate with slippage," European Journal of Mechanics-B/ Fluids, vol. 72, pp. 135-143, 2018.

[16] K. V. Prasad, K. Vajravelu, and H. Vaidya, "Hall effect on MHD flow and heat transfer over a stretching sheet with variable thickness," International Journal for Computational Methods in Engineering Science and Mechanics, vol. 17, no. 4, pp. 288-297, 2016.

[17] P. C. Ram, "Hall effects on hydromagnetic convective flow in a rotating fluid through porous medium," Journal of Engineering Science, vol. 5, pp. 43-53, 1991.

[18] P. Dulal and T. Babulal, "Influence of Hall current and thermal radiation on MHD convective heat and mass transfer in a rotating porous channel with chemical reaction," International Journal of Engineering Mathematics, pp. 2013-13, 2013.

[19] M. Gradeck, B. Hoareau, and M. Lebouché, "Local analysis of heat transfer inside corrugated channel," International Journal of Heat and Mass Transfer, vol. 48, no. 10, pp. 1909-1915, 2005.

[20] C. Bhaskar, D. Sanatan, and R. J. Rabindra, "The Effects of Hall currents and radiation on MHD free convection of a viscous incompressible fluid confined between two vertical walls in a rotating," Advances in Applied Science Research, vol. 3, pp. 3766-3787, 2012.

[21] D. Mahendar and P. S. Rao, "The effect of hall current on an unsteady MHD free convective flow along a vertical porous plate with thermal radiation," IOSR Journal of Mathematics, vol. 11, pp. 122-141, 2015.

[22] M. Kinyanjui, J. K. Kwanza, and S. M. Uppal, "MHD free convection heat and mass transfer of a heat generating fluid past an impulsively started infinite vertical porous plate with Hall current and radiation absorption," Energy Conversion and Management, vol. 42, no. 8, pp. 17-931, 2001.

[23] E. M. AboEldahab, "Convective heat transfer, by the presence of radiation, in an electrically conducting fluid at a stretching surface," Canadian Journal of Physics, vol. 79, no. 6, pp. 929-937, 2001.
[24] K. Giterere, M. Kinyanjui, and S. M. Uppal, "MHD flow in porous media over a stretching surface in rotating system with heat and mass transfer," International Electronic Journal of Pure and Applied Mathematics, vol. 4, no. 1, pp. 9-32, 2012.

[25] R. C. Meyer, "On reducing aerodynamic heat-transfer rates by magnetohydrodynamic techniques," Journal of the Aerospace Sciences, vol. 25, no. 9, pp. 561-566, 1958.

[26] N. Ida, "Faraday's law and induction," in Engineering Electromagnetics, Springer, New York, NY, USA, 2000.

[27] M. Q. Brewster, Thermal Radiative Transfer and Properties, John Wiley \& Sons, New York, NY, USA, 1992.

[28] W. M. Rohsenow, J. P. Hartnett, and Y. I. Cho, Handbook of Heat Transfer, McGraw-Hill, New York, NY, USA, 3rd edition, 1998. 